\title{
CARACTERIZACIÓN MORFOLÓGICA DEL CARIOTIPO DE Guadua angusti- folia Kunth Var. angustifolia. (BAMBUSOIDE: POACEAE)
}

\section{MORPHOLOGICAL CHARACTERIZATION OF KARYOTYPE FOR Guadua angustifolia Kunth Var. angustifolia. (BAMBUSOIDE: POACEAE)}

Jenny Valencia ${ }^{1}$, Nohra Rodríguez ${ }^{1}$, Juan Carlos Herrera² y Gloria Camacho²

\author{
${ }^{1}$ Programa de Biologia Universidad del Quindio. jennycarolina1988@gmail.com, \\ nohrarodriguez@gmail.com \\ ${ }^{2}$ Centro Nacional de investigaciones de café CENICAFE, Disciplina Mejoramiento Genético / Coffee \\ Breeding Program. j.herrera@cafedecolombia.com, cenicafe $@$ cafedecolombia.com.

\section{RESUMEN}

Guadua angustifolia presenta dificultades en su clasificación taxonómica, algunos autores han señalado que dentro de la especie se pueden identificar cuatro variedades, por esta razón el objetivo del presente trabajo fue caracterizar su cariotipo para contribuir al esclarecimiento taxonómico. Para este fin, se evaluó la morfología cromosómica de esta especie a partir de tejido meristemático radicular. Los resultados mostraron que la dotación cromosómica es igual $2 \mathrm{n}=2 \mathrm{X}=46$, Con un valor de $\mathrm{X}=23$, siendo este el primer reporte citogenético para la especie. La caracterización morfológica de los cromosomas permitió establecer la presencia de 4 categorías cromosómicas siendo la metacéntrica la más frecuente (39\% del cariotipo). Se propone clasificar los cromosomas según su tamaño en 5 categorías relativas que oscilan entre 0,6 y 1,5um de longitud. Estas observaciones coinciden con reportes previos sobre el cariotipo de la especie, la cual es considerada como un diploide funcional.

Palabras clave: Citogenética, Cariotipificación, Cromosomas, Índice de Asimetría.

\begin{abstract}
Guadua angustifolia has economic and ecological importance in the regions where it is native and in those where it has been introduced. In the present study was to study morphology chromosome from root meristem tissue. The results showed that the chromosome number is equal to $2 n=2 X=46$, with $X=23$. This is the first cytogenetic report for the specie. Morphological characterization of chromosomes allowed us to establish the presence of 4 categories metacentric chromosome being the most frequent (39\% of the karyotype). It is proposed to classify the chromosomes according to size in 5 categories ranging for 0,6 to 1,5 um long. These observations are consistent with previous reports on the karyotype of the species, which is considered as a functional diploid.
\end{abstract}

Key words: cytogenetic, Karyotipification, Chromosome, Asymmetry index. 


\section{INTRODUCCIÓN}

La especie Guadua angustifolia es uno de los bambúes americanos con importancia a nivel mundial. Es el tercer bambú más alto del mundo superado únicamente por dos especies asiáticas (1). Está distribuido a través de Colombia, Ecuador y Venezuela (2) y ha sido introducido en varios países de Centro América, el Caribe y Asia. En Colombia, G. angustifolia se encuentra desde los 40 hasta los 2400 msnm. Según la Sociedad Colombiana del Bambú esta especie se encuentra distribuida a lo largo de la cordillera central, en los departamentos de Antioquia, Cauca, Caldas, Cundinamarca, Huila, Quindío, Risaralda, Tolima y Valle del Cauca; sin embargo, los rodales más desarrollados se encuentran especialmente en los bosques húmedos y muy húmedos, encontrándose en mayor número en las laderas de montaña, cerca a fuentes hídricas; mientras que en el departamento del Quindío, el 90\% de los guaduales se encuentra en zonas planas o en ondulaciones suaves (1).

Según Sepúlveda et al. (3) las agregaciones de G. angustifolia son una cobertura que presenta buenas características físicas para el suelo, confiriéndole condiciones de estabilidad moderada; facilitando la movilidad del agua por tener una buena porosidad, favoreciendo la conductividad hidráulica y la capacidad de almacenamiento de agua en el suelo; además, existe una reducción considerable en la pérdida de suelo y nutrientes por erosión en comparación con otras coberturas vegetales. Por tal motivo, esta especie posee un alto potencial como regulador hídrico en las microcuencas y como cobertura del suelo, minimizando la cantidad de sedimentos que pueden llegar a las fuentes hídricas, que ocasionan contaminación por aumento de sólidos y nutrientes. Adicionalmente, los rodales de esta especie actúan como sumideros de carbono, producen oxígeno, aportan materia orgánica, conservan la biodiversidad y embellecen el paisaje $(1,4)$.

A pesar de la importancia económica y ecológica de la Guadua, la información citológica de la especie es poca, el número cromosómico, o el cariotipo; la falta de información en estos aspectos es una limitante para establecer una buena clasificación taxonómica y para el uso sostenible de la especie, dado que el cariotipo permite la caracterización de especies, poblaciones, individuos, biotipos o razas (5). La mayoría de documentos retoman la información existente en la subfamilia Bambusoideae para la cual se reportan amplias variaciones cromosómicas que van desde 2 n=46 hasta 96 cromoso- mas, con números básicos que oscilan entre $X=6$ hasta 14 (6).

Por consiguiente, es necesario ampliar la información citogenética dentro de la especie, para contribuir con ello al estudio de la diversidad genética en G. angustifolia. En estudios previos se ha valorado la diversidad con técnicas moleculares empleando marcadores como SSR, RAPDs y AFLPs, sin embargo, los índices de diversidad que presentan estas investigaciones son ambiguos, ya que, empleando AFLPs determina que la diversidad genética es baja y empleando SSRs se determina que es alta(2,7). Por lo tanto, la información citológica llega a ser valiosa debido a que el estudio del número y morfología cromosómica en algunos casos, muestran los cambios ocurridos a través de la historia evolutiva de las especies, que se ve reflejado tanto en el genoma como en el fenotipo (8).

El objetivo del presente estudio se orientó hacia el conocimiento del cariotipo de la especie Guadua angustifolia var. angustifolia, aportando información sobre la forma, el numero y el tamaño de sus cromosomas.

\section{MATERIALES Y MÉTODOS}

\section{Colecta de material vegetal}

Se colectaron 60 chusquínes (nombre que reciben los propágulos obtenidas por propagación vegetativa) y 60 riendas laterales (esquejes) de Guadua angustifolia var. angustifolia biotipo macana en la Reserva Natural "La Montaña del Ocaso" ubicada en el municipio de Quimbaya; fue determinada taxonómicamente (9). Posteriormente éstos fueron transportados hasta el invernadero en una nevera de icopor, cubiertos de papel periódico y en bolsas para evitar su rápida deshidratación. Luego, los propágulos se sembraron en suelo abonado con cascarilla de arroz bajo condiciones de invernadero, realizando riegos periódicamente.

\section{Fase de laboratorio}

El presente trabajo se llevó a cabo en el laboratorio de Biotecnología Vegetal del CIBUQ de la Universidad del Quindío, con la colaboración del laboratorio de Citogenética del Centro Nacional de Investigaciones de Café (CENICAFÉ).

Para obtener tejido radicular apto para este estudio se cortaron ápices radiculares de $1,5 \mathrm{~cm}$ de largo y fueron puestas en colchicina al 1\% para inhibir la mitosis y acumular células en metafase, proceso que se llevo a cabo durante dos horas a temperatura ambiente. A con- 
tinuación fueron fijadas embebiéndolas en una solución de carnoy frio ( 1 acido acético: 3 etanol) durante 24 horas a $3^{\circ} \mathrm{C}$. Para la obtención de los cromosomas metafásicos se trabajó con una doble hidrólisis; la primera parte con acido clorhídrico al $2 \mathrm{~N}$ durante 20 minutos, tres lavados con buffer fosfato salino $\mathrm{pH} 5.0$ y consecutivamente un coctel enzimático de celulasa 3

$6 \% 6 \%$ y macerozima $0,4 \%$ durante 2 horas. La tinción de los cromosomas fue realizada con orceína acética $1 \%$ o DAPI según fuese el caso.

\section{Determinación del número y morfología cromosó- mica}

El conteo del número cromosómico y la determinación del cariotipo de Guadua angustifolia var. angustifolia se realizó a partir del análisis de las 10 mejores metafases, las cuales fueron fotografiadas. Estas microfotografía se obtuvieron en el Centro Nacional de Investigaciones de Café (CENICAFE) sede Chinchina - Caldas en la unidad de mejoramiento genético y biotecnología, en el laboratorio de citogenética haciendo uso de un microscopio óptico de contraste de fases marca Nikon modelo eclipse 90i con cámara VDS vösskohler adaptada. Las imágenes para el conteo cromosómico fueron analizadas con el software LUCIA (2006), el cual es especializado para citogenética y trabaja bajo la plataforma Windows XP.

La imagen que ofreció una mejor dispersión y condensación de los cromosomas se utilizó para la identificación y descripción morfológica, de acuerdo con el ta- maño relativo de cada cromosoma (11), los cuales se clasificaron teniendo en cuenta la posición relativa del centrómero, según las normas propuestas por Levan et al., donde la relación entre brazos $\mathrm{Q} / \mathrm{P}$ determina la siguiente clasificación: $1-1,7 \mu \mathrm{m}=$ metacéntrico $; 1,71-3$ $\mu \mathrm{m}=$ submetacéntrico; 3,1-7 $\mu \mathrm{m}=$ subtelocéntrico $\quad \mathrm{y}$ 7,1- $\infty \mu \mathrm{m}=$ acrocéntrico (12). Algunas de las microfotografías fueron tomadas en contraste de fases, permitiendo la observación de los cromosomas sin el uso de colorante, así el material genético logra verse con claridad y puede realizarse el conteo.

\section{Asimetría cromosómica}

Para la determinación de la asimetría cromosómica se utilizó la metodología propuesta por Romero - Zarco (13); que consiste en la aplicación de la siguiente ecuación:

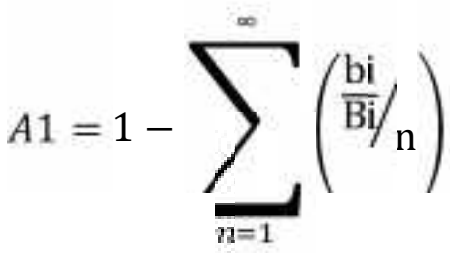

Donde:

A1 = índice de asimetría intracromosomal (maneja rangos de 0 a 1 )

$\mathrm{n}=$ es el numero de cromosomas homólogos

bi $=$ promedio de los brazos cortos de los homólogos

$\mathrm{Bi}=$ promedio de los brazos largos de los homólogos

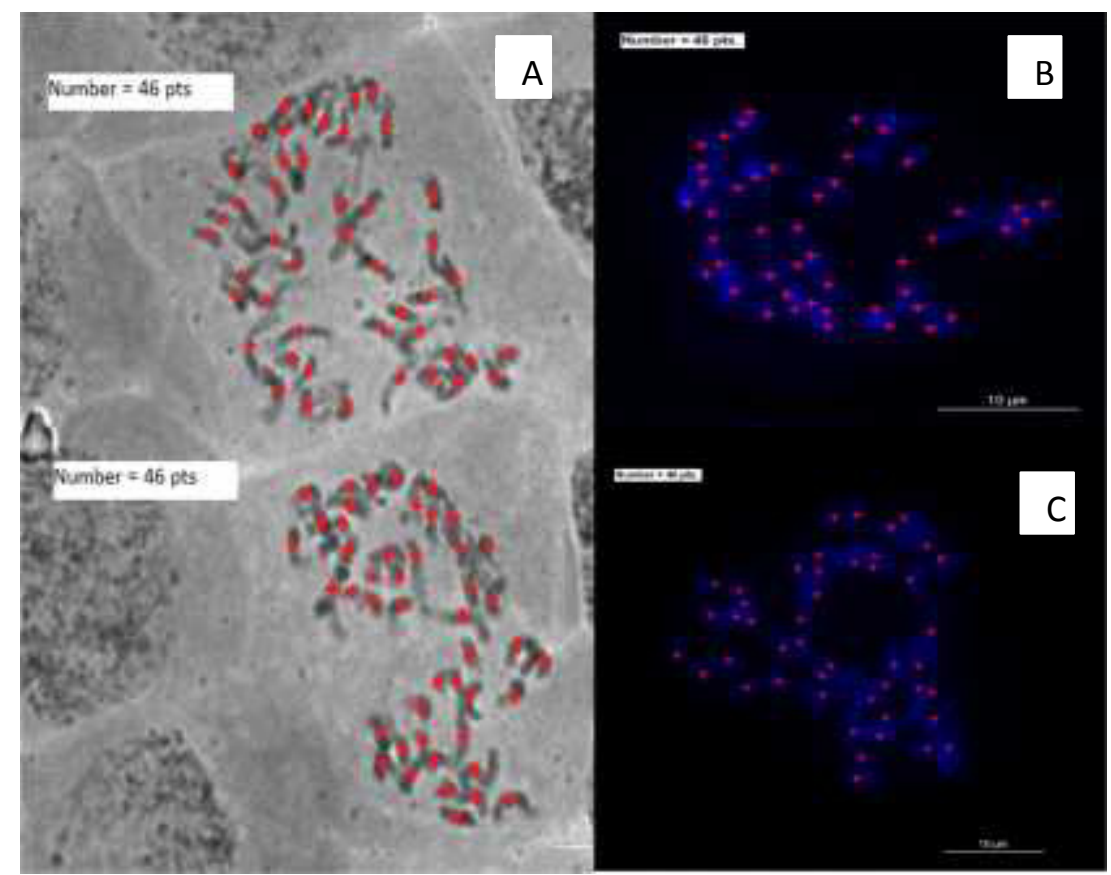

Figura 1. Microfotografías de cromosomas metafásicos de G. angustifolia var. angustifolia A. en contraste de fases. B y C Tinción con DAPI, conteo cromosómico realizado con el Software LUCIA. Campo visual 100X. 
Teniendo como regla de decisión que los valores cercanos a cero indican que los cromosomas del cariotipo estudiados tienden a ser metacéntricos o simétricos.

\section{RESULTADOS}

Los resultados del conteo para G. angustifolia var. angustifolia indicaron que el número cromosómico es $2 \mathrm{n}=2 \mathrm{X}$ $=46$ (Figura 1).

Los 46 cromosomas se organizaron en 5 grupos de tamaño relativo, los cuales varían entre 1,5 a 0,6 $\mu$ m (Tabla 1), organizados en el cariotipo y el ideograma correspondiente a la especie Guadua angustifolia var. angustifolia ( Figura 2).

Tabla 1. Mediciones relativas de los cromosomas de G. angustifolia se detallan las longitudes de las 23 parejas cromosómicas

\begin{tabular}{|c|c|c|c|c|c|c|}
\hline \multirow[t]{2}{*}{ Grupo } & \multirow{2}{*}{$\begin{array}{c}\text { Pareja } \\
\text { Cromosómica }\end{array}$} & \multirow{2}{*}{$\begin{array}{l}\text { Tamaño } \\
\text { Relativo } \\
\text { (Aprox.) }\end{array}$} & \multicolumn{2}{|c|}{ Tamaño Relativo } & \multirow{2}{*}{$\begin{array}{l}\text { Relación de } \\
\text { los brazos } \\
\text { (Q/P) }\end{array}$} & \multirow[t]{2}{*}{ Clasificación } \\
\hline & & & $P$ & $Q$ & & \\
\hline \multirow[t]{5}{*}{ I } & 1 & $1,5 \mu \mathrm{m}$ & $0,4 \mu \mathrm{m}$ & $1,1 \mu \mathrm{m}$ & 2,75 & submetacéntrico \\
\hline & 2 & $1,2 \mu \mathrm{m}$ & $0,6 \mu \mathrm{m}$ & $0,6 \mu \mathrm{m}$ & 1 & metacéntrico \\
\hline & 3 & $1,3 \mu \mathrm{m}$ & $0,5 \mu \mathrm{m}$ & $0,8 \mu \mathrm{m}$ & 1,6 & metacéntrico \\
\hline & 4 & $1,3 \mu \mathrm{m}$ & $0,5 \mu \mathrm{m}$ & $0,8 \mu \mathrm{m}$ & 1,6 & metacéntrico \\
\hline & 5 & $1,2 \mu \mathrm{m}$ & $0,5 \mu \mathrm{m}$ & $0,7 \mu \mathrm{m}$ & 1,4 & metacéntrico \\
\hline \multirow[t]{7}{*}{ II } & 6 & $1,2 \mu \mathrm{m}$ & $0,5 \mu \mathrm{m}$ & $0,8 \mu \mathrm{m}$ & 1,4 & metacéntrico \\
\hline & 7 & $1,1 \mu \mathrm{m}$ & $0,4 \mu \mathrm{m}$ & $0,7 \mu \mathrm{m}$ & 1,7 & metacéntrico \\
\hline & 8 & $1,3 \mu \mathrm{m}$ & $0,3 \mu \mathrm{m}$ & $1 \mu \mathrm{m}$ & 3 & submetacéntrico \\
\hline & 9 & $1,3 \mu \mathrm{m}$ & $0,1 \mu \mathrm{m}$ & $1,2 \mu \mathrm{m}$ & 12 & acrocéntrico \\
\hline & 10 & $1,2 \mu \mathrm{m}$ & $0,1 \mu \mathrm{m}$ & $1,1 \mu \mathrm{m}$ & 11 & acrocéntrico \\
\hline & 11 & $1,1 \mu \mathrm{m}$ & $0,1 \mu \mathrm{m}$ & $1 \mu \mathrm{m}$ & 10 & acrocéntrico \\
\hline & 12 & $0,9 \mu \mathrm{m}$ & $0,4 \mu \mathrm{m}$ & $0,5 \mu \mathrm{m}$ & 1,25 & metacéntrico \\
\hline \multirow[t]{6}{*}{ III } & 13 & $1 \mu \mathrm{m}$ & $0,3 \mu \mathrm{m}$ & $0,7 \mu \mathrm{m}$ & 2,3 & submetacéntrico \\
\hline & 14 & $1 \mu \mathrm{m}$ & $0,1 \mu \mathrm{m}$ & $0,9 \mu \mathrm{m}$ & 9 & acrocéntrico \\
\hline & 15 & $0,9 \mu \mathrm{m}$ & $0,1 \mu \mathrm{m}$ & $0,8 \mu \mathrm{m}$ & 8 & acrocéntrico \\
\hline & 16 & $0,8 \mu \mathrm{m}$ & $0,4 \mu \mathrm{m}$ & $0,4 \mu \mathrm{m}$ & 1 & metacéntrico \\
\hline & 17 & $0,7 \mu \mathrm{m}$ & $0,3 \mu \mathrm{m}$ & $0,4 \mu \mathrm{m}$ & 1,3 & metacéntrico \\
\hline & 18 & $0,7 \mu \mathrm{m}$ & $0,1 \mu \mathrm{m}$ & $0,6 \mu \mathrm{m}$ & 6 & subtelocéntrico \\
\hline \multirow[t]{3}{*}{ IV } & 19 & $0,7 \mu \mathrm{m}$ & $0,3 \mu \mathrm{m}$ & $0,4 \mu \mathrm{m}$ & 6 & subtelocéntrico \\
\hline & 20 & $0,8 \mu \mathrm{m}$ & $0,1 \mu \mathrm{m}$ & $0,7 \mu \mathrm{m}$ & 7 & subtelocéntrico \\
\hline & 21 & $0,8 \mu \mathrm{m}$ & $0,1 \mu \mathrm{m}$ & $0,7 \mu \mathrm{m}$ & 7 & subtelocéntrico \\
\hline \multirow[t]{2}{*}{$\mathbf{v}$} & 22 & $0,6 \mu \mathrm{m}$ & $0,1 \mu \mathrm{m}$ & $0,5 \mu \mathrm{m}$ & 5 & subtelocéntrico \\
\hline & 23 & $0,6 \mu \mathrm{m}$ & $0,1 \mu \mathrm{m}$ & $0,5 \mu \mathrm{m}$ & 5 & subtelocéntrico \\
\hline
\end{tabular}




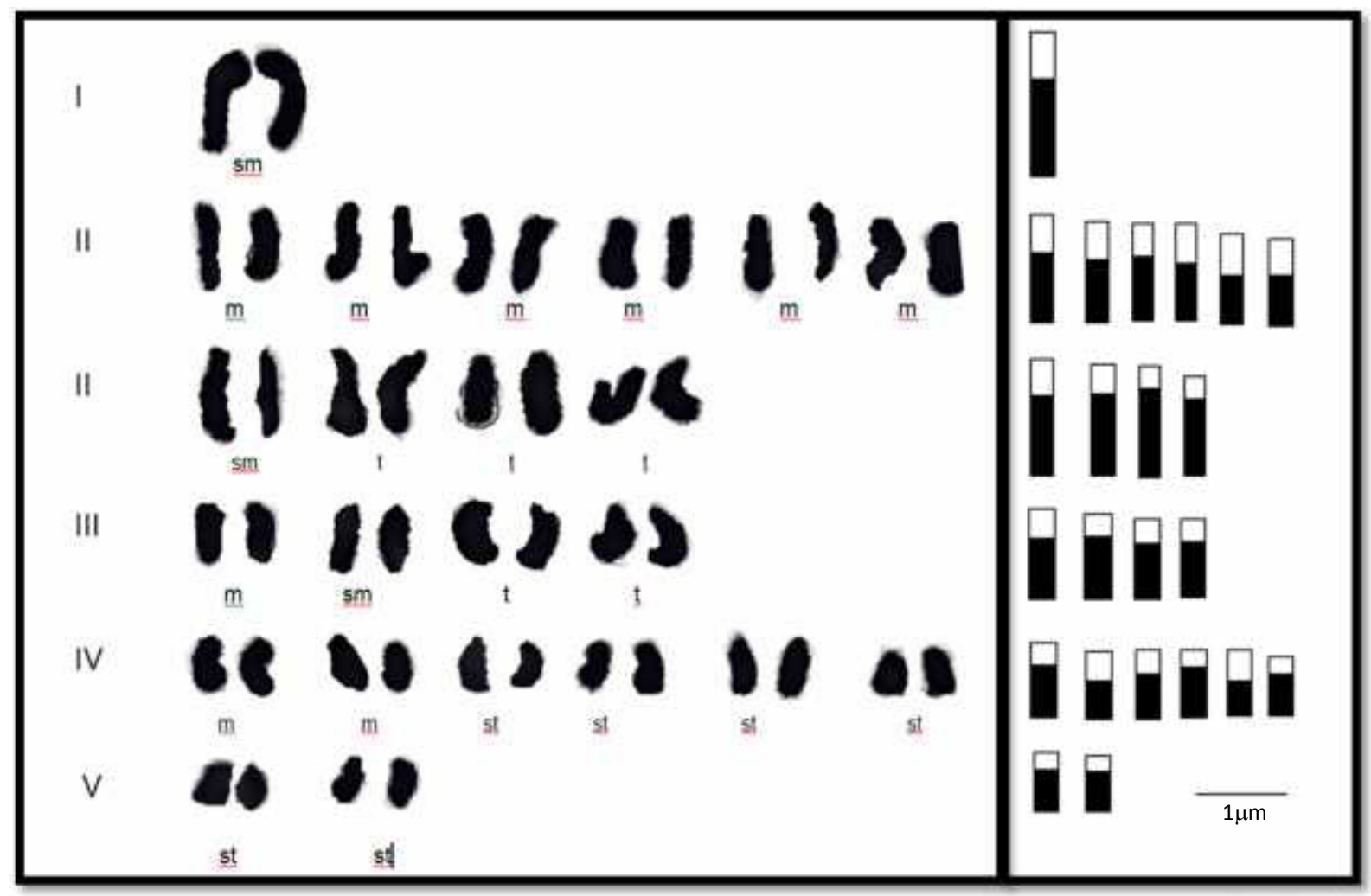

Figura 5. Cariotipo e ideograma de Guadua angustifolia var. angustifolia biotipo macana, según la posición relativa del centrómero $(100 \mathrm{X})$.

\section{DISCUSIÓN}

La dotación cromosómica encontrada para G. angustifolia var. angustifolia coincide con los valores reportados para tres especies de bambúes leñosos dentro del género estás son: Guadua capitata (14), Guadua chacoensis $(15,16)$, Guadua paraguayana (15), los cuales reportan valores de $2 \mathrm{n}=2 \mathrm{x}=46$.

Estos resultados confirman que G. angustifolia var. angustifolia es un bambú leñoso diploide. Con base en estas observaciones se propone por primera vez un cariotipo y un ideograma para la especie a partir de datos morfológicos como la posición relativa del centrómero y el tamaño relativo de los brazos cromosómicos (Figura 2).

El cariotipo de G. angustifolia var, angustifolia está conformado por 23 pares de cromosomas de los cuales el $39 \%$ son metacéntricos, el $26 \%$ subtelocéntricos, el 22\% acrocéntricos y el 13\% submetacéntricos. Con base en los parámetros propuestos por Fukui y Nakayama (10) la especie estudiada posee cromosomas pequeños, ya que, su tamaño relativo se encuentra entre $<1$ - $3 \mu \mathrm{m}$ (Tabla 1), lo que es común dentro de la familia Poaceae. Stebbins (16) propone la hipótesis que las plantas primitivas presentan cromosomas grandes, mientras que las plantas evolutivamente recientes poseen cromosomas pequeños, ciclos de vida reducidos y concentración de regiones de heterocromatina dentro de los centrómeros, Dicha hipótesis podría ser aplicable al caso del genero Guadua dado que el origen de la familia a la cual pertenece esta especie, se remonta a 70 a 55 millones de años atrás (17); además, que es una especie de distribución restringida al norte de Sur América en especial a los Andes, los cuales se consideran formaciones derivadas de eventos geológicos recientes. Se puede observar que un alto porcentaje de los cromosomas es metacéntrico (39\%), siendo además los cromosomas de mayor tamaño, y también se observa un alto porcentaje $(61 \%)$ de cromosomas que probablemente se derivaron de las formas ancestrales metacéntricas y formaron cromosomas de menor tamaño que adicionalmente modificaron la posición del centrómero, siendo estas características de los cariotipos de plantas mas recientes.

Las modificaciones en el tamaño cromosómico y su posición centromérica, así como la determinación del número cromosómico es una parte integral del estudio de la sistemática en plantas; los estudios de cariotipo y tamaño cromosómico son poco comunes. Sin embargo, 
el cariotipo y número cromosómico cambia a través de la historia evolutiva de los grupos taxonómicos; por lo tanto, se ha visto que en los últimos años la evaluación de la evolución cromosómica de los grupos ha enriquecido los datos filogenéticos (8) Kellogg (17) propone que el grupo de las gramíneas es monofilético, considerando además, que el número cromosómico es un carácter estable dentro de las subfamilias y es usado como sinapomorfía para la definición de dos de las agrupaciones en el cladograma; lo que concuerda con lo encontrado en este trabajo. La dotación cromosómica para la variedad angustifolia fue $2 n=2 x=46$, y para el género Guadua se reportan $2 \mathrm{n}=2 \mathrm{x}=46$ a 48 (6), lo que podría emplearse como evidencia de su origen monofilético. Según el índice de asimetría intracromosomal $(0,984)$ el cariotipo para la especie es asimétrico, a pesar que el mayor porcentaje de cromosomas es metacéntrico. Teniendo en cuenta esto, Stebings (16) plantea que en plantas superiores, los cambios en los caracteres morfológicos de los cromosomas tienden a estar relacionados con la evolución del grupo en estudio; además, sugiere una tendencia general que indica que la asimetría ca- riotípica se asocia con la adaptación a diferentes ambientes, En el caso particular de las Poaceae el autor sugiere, que su variación en el tamaño cromosómico ésta relacionado con las adaptaciones al hábitat; ya que, plantas de clima templado presentan cromosomas grandes, mientras que los cromosomas pequeños y medianos son predominantemente de plantas con distribución tropical o subtropical coincidiendo con lo encontrado en este trabajo, ya que esta es una especie endémica del neotrópico.

Se recomienda en posteriores estudios hacer bandeos cromosómicos para la identificación de rearreglos o de incrementos en el nivel de ploidía, como mecanismos de especiación.

\section{CONCLUSIÓN}

Guadua angustifolia var, angustifolia es diploide con $2 \mathrm{n}=46$, presenta cromosomas de tamaño pequeño que oscilan entre $1,5 \mu \mathrm{m}$ y $0,6 \mu \mathrm{m}$, y un índice de asimetría intracromosomal de 0,984 , características que sugieren que la especie presenta cambios evolutivos recientes.

\section{BIBLIOGRAFÍA}

1. Cruz H, Bambú - Guadua, Guadua angustifolia Kunt,- Bosques Naturales en Colombia y plantaciones comerciales en México, 1era edición, Grafica olímpicas S,A, 2009,

2. Marulanda M, López A, y Claroz J, Analyzing the genetic diversity of Guadua spp, In Colombia using rice and sugarcane microsatellites, In: Crop Breed and Applied Biotechnology, Brazilian Society of Plant Breeding, 2007 ; (7): 43-51,

3. Sepúlveda I, Rodríguez J, y Camargo J, Beneficios Ambientales de la Guadua angustifolia Kunth en la Protección del Suelo en el Eje Cafetero colombiano, memorias simposio internacional de guadua, Pereira-Colombia, 2004,

4. Cruz H, La Guadua nuestro bambú, Corporación autónoma regional del Quindío - centro nacional para el estudio del bambú Guadua, 1994; 34 - 121p,

5. Bandini R, Filogeografia, diferenciaçāo geográfica e caracterizaçāo citogenética de populaçōes naturais de Psychotria ipecacuana das florestas atlántica e amazŏnica, Viçosia - Minas Gerais; Brasil, 2007

6. Andrada A, Lozzia M, y Cristobal E, Contribución al conocimiento citológico de Guadua chacoensis, Rev, Lilloa, 2007; 44 (1-2): 3-6,

7. Potosí C,; Vallejo F, y Palacio J, Estimación mediante RAPD's de la diversidad genética en Guadua en el departamento del Cauca, Colombia, Tesis de Maestría en Recursos Fitogenéticos Neotropicales, Universidad Nacional de Colombia Sede Palmira, 2006,

8. Crawford D, Mort M, and Archibald J, Biosystematic, Chromosomes and molecular data: Melding the old an the new, Taxon, 2005; 54(2):285-289,

9. Fukui F, y Nakayama S, Plant Chromosomes, Laboratory Methods, CRC Press Inc, New York, 1996; $274 \mathrm{p}$,

10. Gómez G, Cruz S,, Agudelo C, y Giraldo E, Caracterización morfológica y anatómica de cuatro biotipos de Guadua angustifolia Kunth, Monografías de la flora andina, Publicaciones seriadas Universidad del Quindío, Herbario Universidad del Quindío, 2001 (3): 119-159p,

11. Singh J, R, Plant cytogenetics, Second edition, Florida, United States, CRC Press, 2003; 463p, 
12. Levan A,, Fredga K, y Sandberg A, Nomenclature for centromeric position on chromosomes, Hereditas 1964; 52: 201- 220,

13. Romero - Zarco C, A new method for estimating karyotype asymetric, Taxon 1986; 35 (3): 526-530,

14. Gould F, y Soderstrom T, Chromosome numbers of tropical American grasses, American Journal of Botany, 1967; 54: 676-683,

15. Quarin C, Recuentos cromosómicos en gramíneas de Argentina subtropical, Hickenia, 1977; 1: 7378 ,

16. Stebbins L, Chromosomal variation and evolution: polyploidy and chromosome size and number shed light on evolutionary processes in higher plants, Science, 1966; 152 (3728): 1463-1469,

17. Kellogg, E, Evolutionary History of the Grasses, Plant Physiology, 2001; 125: 1198-1200, 